\title{
Le bilan scientifique de la mission Megha-Tropiques après 8 ans dans l'espace
}

\author{
Rémy Roca ${ }^{1}$, Philippe Chambon ${ }^{2}$, Marielle Gosset ${ }^{3}$, \\ Dominique Bouniol ${ }^{2}$, Hélène Brogniez ${ }^{4}$, Sophie Cloché ${ }^{5}$, \\ Thomas Fiolleau', Patrick Raberanto ${ }^{6}$, Michel Dejus ${ }^{7}$ \\ 1. Laboratoire d'études en géophysique et océanographie spatiales, \\ Université Paul Sabatier / CNRS / Cnes / IRD, Toulouse \\ 2. Centre national de recherches météorologiques, Université de Toulouse/ \\ Météo-France / CNRS / Toulouse \\ 3. Géoscience Environnement Toulouse, Université Paul Sabatier / CNRS / IRD, \\ Toulouse \\ 4. Laboratoire atmosphères, milieux, observations spatiales, \\ Institut Pierre-Simon Laplace, UVSQ / Sorbonne Université / CNRS, Guyancourt \\ 5. Institut Pierre-Simon Laplace, École Polytechnique / CNRS, Palaiseau \\ 6. Laboratoire de météorologie dynamique, École Polytechnique / CNRS, Palaiseau \\ 7. Cnes, Toulouse
}

remy.roca@legos.obs-mip.fr

\section{Résumé}

La mission Megha-Tropiques récolte des observations du cycle de l'eau et de l'énergie dans l'atmosphère intertropicale depuis 2011. L'utilisation de ces observations a démontré la pertinence de son orbite originale inclinée sur l'équateur, tant pour l'estimation des précipitations que pour la prévision numérique du temps. L'exploitation scientifique, toujours en cours, autour de l'étude des relations entre la vapeur d'eau et les nuages, le cycle de vie des systèmes convectifs tropicaux et l'hydrométéorologie a permis plusieurs avancées brièvement présentées ici. De nombreux centres opérationnels à travers le monde assimilent les données de la mission, disponibles en temps réel, grâce aux récents progrès de l'interprétation de la mesure à $183 \mathrm{GHz}$. es régions tropicales $\left(30^{\circ} \mathrm{S}-30^{\circ} \mathrm{N}\right)$ sont associées à un cycle de l'eau et de l'énergie très intense dont les retentissements s'expriment à l'échelle du climat de la planète entière. La convection y est organisée sous la forme de systèmes convectifs qui redistribuent l'excès d'énergie et d'humidité dans l'atmosphère et sur les surfaces continentales via des processus encore mal compris et difficiles à représenter dans les modèles de climat. Le réchauffement global perturbe fortement les quantités d'énergie et d'eau mises en jeu dans le climat des tropiques. L'évolution future de leurs distributions reste très incertaine. Elle fait l'objet d'intenses recherches coordonnées par le Programme mondial de recherche sur le climat (PMRC) et en particulier la communauté organisée autour du projet Global Energy and Water Exchanges (Gewex).

Les réseaux d'observations conventionnelles sont particulièrement épars aux tropiques (sans mentionner les régions océaniques) et ne permettent pas une caractérisation précise de l'eau atmosphérique et des flux radiatifs. L'observation depuis l'espace offre une véritable solution alternative pour décrire l'environnement dans ces régions. Dans la foulée de la Tropical Rainfall Measuring Mission de la Nasa et de l'Agence d'exploration aérospatiale japonaise (Jaxa), la France et l'Inde ont initié voilà plus de 20 ans une coopération scientifique et technologique pour contribuer à l'étude du cycle de l'eau et de l'énergie des régions tropicales : la mission Megha-Tropiques (Desbois et al., 2007). Alors que Megha signifie nuages en sanskrit et rappelle le cycle de l'eau atmosphérique, Tropiques renvoie à une orbite originale dédiée à l'observation de la ceinture intertropicale. L'orbite de la mission est détaillée dans Capderou (2017). Le satellite a été réalisé conjointement par l'Organisation de la recherche spatiale indienne (Isro) et le Centre national d'études spatiales (Cnes) et délivre des données depuis fin 2011 renforçant ainsi le système global d'observations et en particulier la constellation internationale Global Precipitation Mission (Hou et al., 2014). 


\section{Abstract}

The scientific assessment of the Megha-Tropiques mission after 8 years in space

The Megha-Tropiques mission has been collecting observations of the water and energy cycle in the intertropical atmosphere since 2011. The use of these observations has demonstrated the relevance of its original orbit inclined over the Equator both for the precipitation estimates and for numerical weather prediction. The ongoing scientific exploration of the relationship between water vapor and clouds, the life cycle of tropical convective systems, and hydrometeorology has led to many advances that are briefly presented here. Many operational centres around the world are assimilating available mission data in real time thanks to recent advances in the interpretation of the $183 \mathrm{GHz}$ measurement.
Tableau 1. Synthèse des caractéristiques des instruments à bord du satellite Megha-Tropiques.

\begin{tabular}{|c|c|c|c|c|}
\hline Instrumer & Canaux & $\begin{array}{l}\text { Résolution sol } \\
\text { (diamètre) }\end{array}$ & $\begin{array}{l}\text { Largeur de } \\
\text { la fauchée }(\mathrm{km})\end{array}$ & Commentaires \\
\hline Madras & $18,7,23,8,36,5,89,0,157,0 \mathrm{GHz}$ & $\begin{array}{l}\text { D'environ } \\
40 \text { km à } 6 \text { km }\end{array}$ & $\sim 1700$ & $\begin{array}{l}\text { Polarisation } \\
\text { horizontale et } \\
\text { verticale }\end{array}$ \\
\hline Saphir & Six canaux autour de $183,31 \mathrm{GHz}$ & $\begin{array}{l}10 \mathrm{~km} \text { au nadir } \\
16 \mathrm{~km} \text { en bord }\end{array}$ & $\sim 1700$ & \\
\hline Scarab & $\begin{array}{l}\text { Canal total : } 0,2 \text { à } 200 \mu \mathrm{m} \\
\text { Canal solaire : } 0,2 \text { à } 4 \mu \mathrm{m} \\
\text { Canal visible : } 0,5 \text { à } 0,7 \mu \mathrm{m} \\
\text { Canal infrarouge thermique : } \\
10,5 \text { à } 12,5 \mu \mathrm{m}\end{array}$ & $\begin{array}{l}50 \mathrm{~km} \text { au nadir } \\
100 \mathrm{~km} \text { en bord }\end{array}$ & $\sim 2200$ & \\
\hline
\end{tabular}

\section{Le satellite et les instruments}

\section{Présentation rapide}

Plusieurs publications présentent en détail la mission et les spécifications instrumentales (Desbois et al., 2007 ; Roca et al., 2015). Le satellite MeghaTropiques embarque à son bord trois instruments de conception française (figure 1). Le premier est un radiomètre imageur micro-ondes multi fréquences à balayage conique Madras ${ }^{1}$ qui est dédié à l'estimation des taux de pluies instantanées, le deuxième est un radiomètre sondeur micro-ondes à balayage perpendiculaire à la trace Saphir ${ }^{2}$ qui opère aux fréquences proches de $183 \mathrm{GHz}$, ce qui permet une description verticale de l'humidité atmosphérique, et le troisième est $\mathrm{Scarab}^{3}$, un radiomètre bandes larges pour la mesure du bilan radiatif au sommet de l'atmosphère. Les spécificités de chaque instrument sont résumées dans le tableau 1 . Notons qu'un récepteur GPS (Rosa) opéré par l'Isro est également à bord de la plateforme. L'orbite quasi circulaire orientée à $20^{\circ}$ sur l'équateur et située à $867 \mathrm{~km}$ d'altitude fournit une forte revisite, qui permet d'observer six fois par jour le même lieu dans les régions intertropicales et incarne la forte innovation amenée par la mission.

Depuis le début des opérations, on distingue trois phases d'exploitation de la mission : la période de recette jusqu'à l'arrêt de Madras (octobre 2011 -janvier 2013), la période d'opération nominale de Saphir et Scarab (octobre 2011-fin 2018) et la période de fonctionnement dégradé (de fin 2018 à nos jours), où seulement $25 \%$ environ des observations sont dorénavant disponibles. Les problèmes

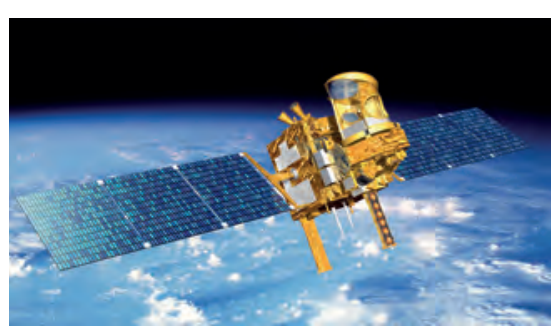

Figure 1. Vue d'artiste du satellite MeghaTropiques. Crédit: Cnes.

techniques de Madras n'ont pas permis l'exploitation scientifique initialement prévue, mais une grande partie des objectifs scientifiques associés à cet instrument a néanmoins été atteinte grâce à l'instrument Saphir ainsi qu'aux progrès récents concernant l'interprétation des scènes associée à la diffusion du rayonnement à $183 \mathrm{GHz}$. C'est donc principalement autour de Saphir et de Scarab que l'exploitation de la mission Megha-Tropiques s'est articulée.

Les deux radiomètres fonctionnent de manière continue depuis le lancement, sans souci majeur, en plein accord avec les spécifications de mission (Karouche et al., 2012). Les performances radiométriques de Saphir ont été reconnues par la communauté internationale et, depuis 2015, Saphir sert d'étalon pour les sondeurs micro-ondes de la constellation Global Precipitation Measurement mission (GPM). La stabilité de leurs performances radiométriques est de première qualité (figure 2). Après plus de 150 millions de révolutions, les données de Saphir sont aussi précises qu'au premier jour !

1. Madras est un acronyme anglais pour "Microwave Analysis and Detection of Rain and Atmosphere Systems ».

2. Saphir vient de "Sondeur atmosphérique du profil d'humidité intertropicale par radiométrie ». 3. Scarab est un acronyme en anglais pour «Scanner for Radiation Budget ». 


\section{La démonstration de faisabilité de la mission}

L'atténuation de la perte de Madras par l'utilisation de Saphir a permis en particulier de finaliser la démonstration de faisabilité de la mission selon deux axes : l'estimation des précipitations et la prévision numérique du temps aux tropiques. Notons que la durée de réalisation de la mission (initiée en 1993 pour un début des opérations en 2013) rend cet exercice très intéressant dans la mesure où, il y a 25 ans, la constellation d'observations micro-ondes était dépeuplée au regard de la période actuelle, les systèmes d'assimilation pour la prévision numérique du temps balbutiaient avec les approches variationnelles quadridimensionnelles et les sondeurs hyperspectraux n'existaient pas encore. Néanmoins, avec l'avènement d'une constellation de plateformes offrant des observations micro-ondes fournies, il a été montré que l'estimation des pluies bénéficie toujours et de manière significative de l'échantillonnage de Megha-Tropiques (Roca et al., 2018). De même, les études d'assimilation des observations de Saphir ont montré un impact important de l'échantillonnage de Megha-Tropiques dans le système d'observations actuel, autrement plus dense qu'il y a vingt ans ! Cette démonstration a permis de convaincre nos collègues de la Nasa, qui intègrent depuis fin 2018 les observations issues
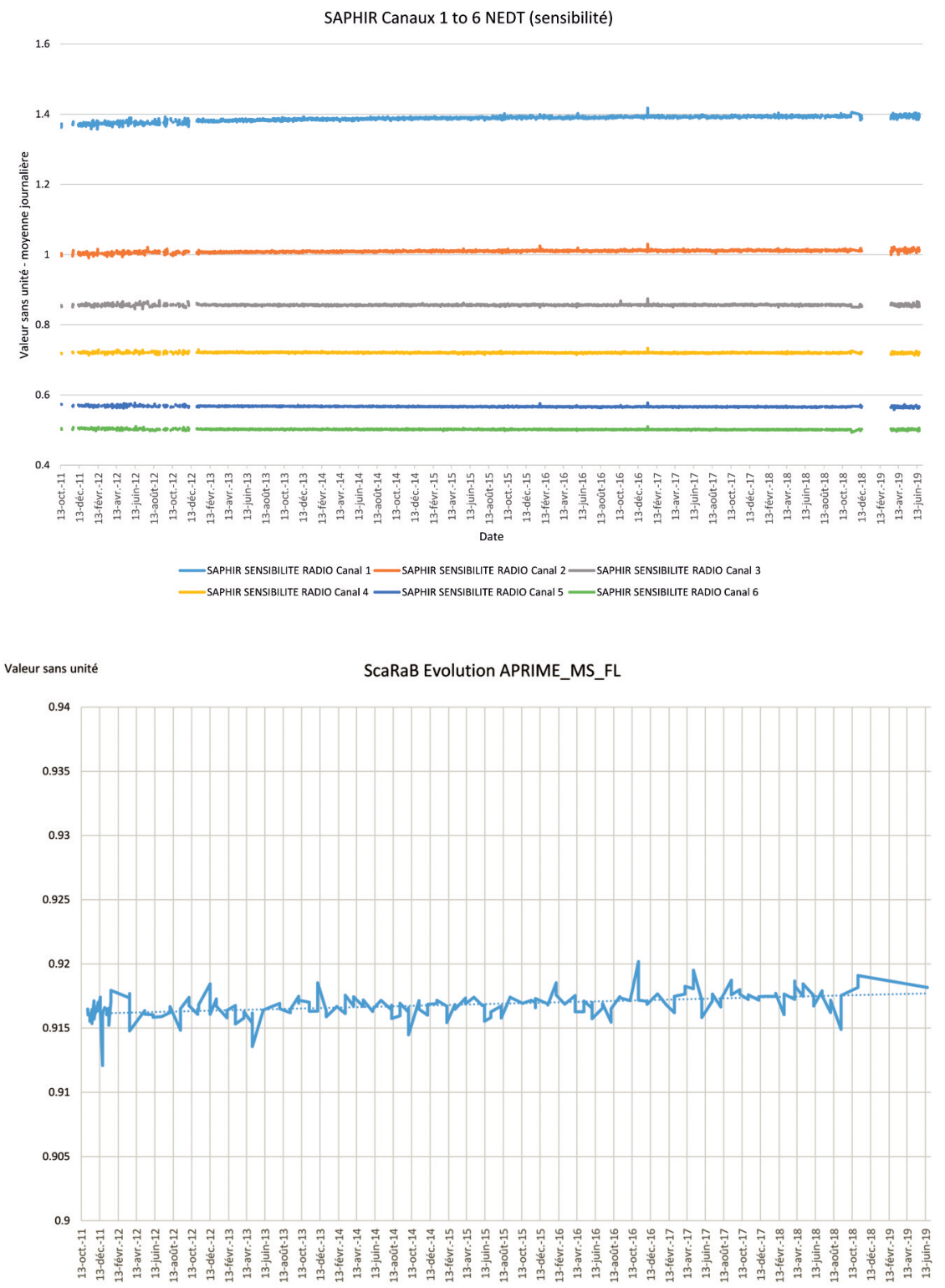

Figure 2. Évolution temporelle des performances instrumentales depuis le début de la mission. En haut, la température équivalent de bruit en $\mathrm{K}$ des six canaux de Saphir ; cette variable donne une bonne indication de la sensibilité de l'instrument et son suivi dans le temps du vieillissement. En bas, Le coefficient $A^{\prime}$, rapport des luminances filtrées du canal " ondes courtes » et du canal total de scènes " ondes courtes" en mode solaire total (MS TOT) permettant de quantifier la dégradation relative des deux canaux.

de Saphir dans leur produit phare d'estimation des précipitations Imerg, et de nombreux centres météorologiques d'assimiler Saphir comme détaillé plus bas.

\section{Les objectifs scientifiques de la mission}

Les observations de la mission Megha-Tropiques nourrissent plusieurs questions scientifiques qui relèvent tant de la physique de l'atmosphère (relation entre les nuages et l'humidité troposphérique), du fonctionnement de la convection organisée (le cycle de vie des systèmes convectifs) ou bien de l'hydrométéorologie tropicale (exploration des relations pluiecrues).

\section{Lien nuages-vapeur d'eau}

Les interactions entre les nuages et l'humidité de leur environnement sont très nombreuses, évoluent fortement au cours du cycle de vie des nuages et impliquent des processus thermodynamiques et dynamiques entremêlés. Une modification de la couverture nuageuse induite par les variations climatiques globales peut être associée à des rétroactions qui dépendent de l'altitude et de la phase des nuages (gouttelettes et/ou cristaux de glace, formés dans des zones de pollution ou non), ainsi que de la surface sous-jacente. Ainsi, selon leurs propriétés macro et microphysiques, les nuages peuvent refroidir ou réchauffer l'atmosphère et la surface. Cette complexité dans les interactions fait du cycle de l'eau atmosphérique un élément de variation interne du climat encore très incertain face aux exercices de projection climatique et constitue ainsi un verrou important qui s'exerce à de multiples échelles spatiales et temporelles. Le design de l'instrument Saphir permet d'étudier la variation verticale de l'humidité. Ainsi, le cycle diurne des phénomènes nuageux et celui de l'humidité environnante peuvent être abordés en exploitant la synergie des mesures d'humidité de l'instrument Saphir avec les mesures des propriétés macrophysiques des nuages, comme celles fournies par le lidar Cats ${ }^{4}$, porté

4. Le lidar Cats (Cloud-Aerosol Transport Systems) est un lidar de la Nasa. 
pendant deux ans (2015-2017) par la Station spatiale internationale (Chepfer et al., 2019). Comme le présente schématiquement la figure 3 , on peut découper la zone tropicale en deux parties selon la circulation de HadleyWalker : une branche d'ascendance, dominée par des mouvements verticaux intenses caractérisant les cellules de convection, et une branche de subsidence, dans laquelle les mouvements convectifs sont beaucoup plus restreints verticalement, ce qui limite l'extension verticale des nuages aux premiers kilomètres. La figure 3 montre aussi l'évolution diurne de l'humidité relative et des nuages plus ou moins épais optiquement dans les zones océaniques de subsidence de grande échelle. On peut ainsi visualiser l'humidification de la basse troposphère (de la surface à environ $4 \mathrm{~km}$ ) autour du lever du soleil qui suit le développement de nuages fins situés en altitude, dont le maximum de développement est atteint plus tard pendant la nuit. On peut voir également l'humidification de la moyenne troposphère (jusqu'à environ $8 \mathrm{~km}$ ) associée à la dissipation des nuages épais qui sont observés sur la quasi-totalité de la troposphère libre (de 2 à $16 \mathrm{~km}$ en altitude) au moment du coucher du soleil. De telles études couplant les capacités d'observation de différents instruments qui ciblent différents aspects du cycle de l'eau atmosphérique sont particulièrement informatives sur les schémas d'évolution des nuages et de leur environnement. Elles apportent ainsi des nouvelles connaissances sur les rétroactions nuages-vapeur d'eau-climat, qui sont entachées de nombreuses incertitudes, alors qu'elles jouent un rôle essentiel dans l'équilibre énergétique de la Terre.

\section{Cycle de vie des systèmes convectifs et rayonnement}

La présence généralisée de systèmes convectifs sous les tropiques est à l'origine de nuages hauts contrôlant très fortement le bilan radiatif de cette région. La fusion des observations du radiomètre Scarab et des observations des satellites géostationnaires a permis une documentation de l'évolution des propriétés radiatives au cours du cycle de vie des systèmes convectifs. En effet, les études antérieures (Houze, 2018) montrent une diversité de processus et une très forte évolution de la taille de ces objets, ce qui nécessite la prise en compte de la dimension temporelle dans leur documentation. L'application systématique de l'algorithme Toocan (Fiolleau et Roca, 2013) à l'ensemble des observations des satellites géostationnaires, une fois homogénéisées, permet un suivi des objets depuis leur initiation jusqu'à leur dissipation.
L'analyse statistique de la base de données ainsi obtenue montre que les systèmes dont la durée de vie excède 5 heures expliquent, à l'échelle des Tropiques, plus de $97,5 \%$ de la nébulosité dont la température dans l'infrarouge est inférieure à $235 \mathrm{~K}$. L'analyse de l'évolution de la taille de la couverture nuageuse associée à ces systèmes de plus de 5 heures permet de mettre en lumière que $85 \%$ d'entre eux atteignent leur taille maximale au milieu de leur cycle de vie en ne connaissant qu'une phase de croissance et de décroissante de son extension (Roca et al., 2017). Cette propriété permet de construire une vision composite du cycle de vie de la nébulosité associée à la convection profonde, où chaque processus peut être positionné par rapport à un cycle de vie normalisé par la durée de vie. L'existence d'un système est donc divisée en dix étapes d'égale durée. Cette approche a été utilisée pour y adjoindre les observations de 1'A-Train ${ }^{5}$ afin de décrire les processus microphysiques mis en jeu au cours de la vie des systèmes convectifs (Bouniol et al., 2016).

5. L'A-Train est une constellation de satellites situés sur la même orbite et qui se suivent de quelques minutes, dont l'un des objectifs est l'observation de la répartition verticale des propriétés nuageuses et du bilan radiatif associé.

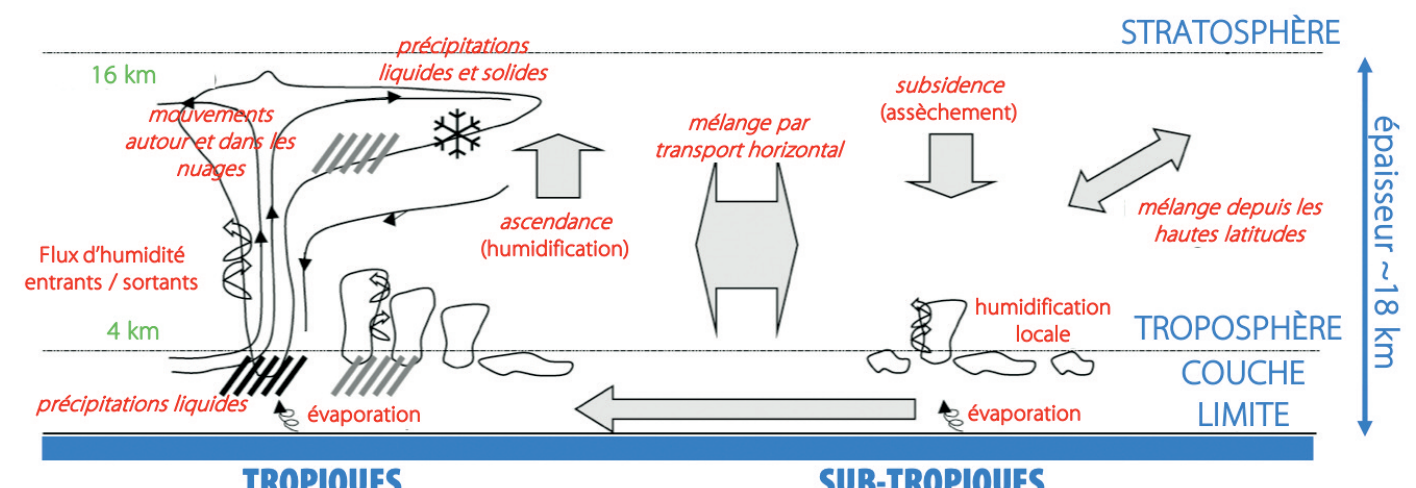

TROPIQUES

Humidité Relative (SAPHIR/MEGHA-TROPIQUES)
Quantité de nuages épais (CATS/ISS)
Quantité de nuages fins (CATS/ISS)
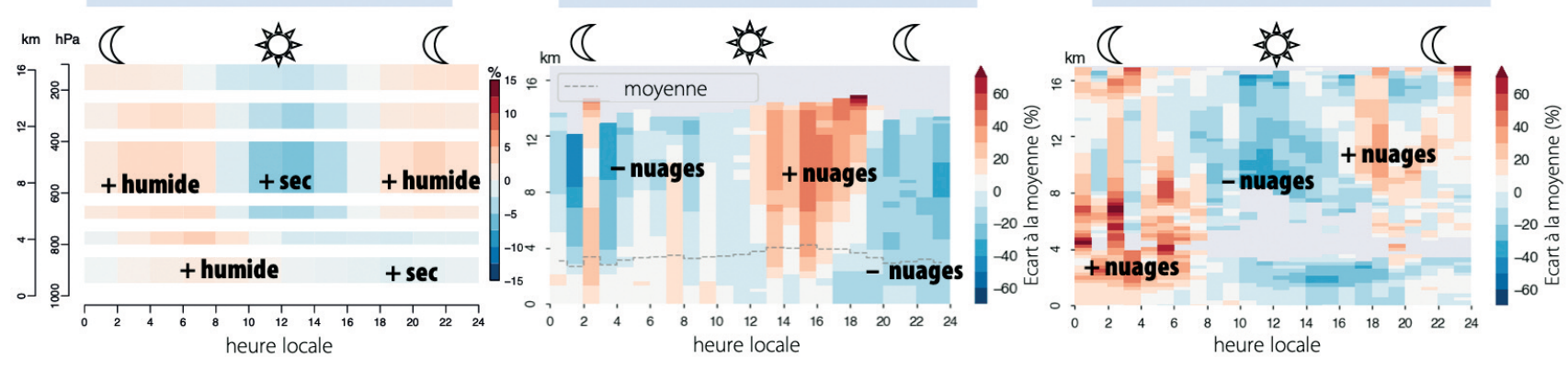

Figure 3. En haut, schéma des processus d'assèchement et d'humidification dans l'atmosphère tropicale (figure modifiée de Sherwood et al., 2010). En bas, évolutions diurnes de l'humidité relative, telle qu'observée par Saphir, et des quantités de nuages épais et fins, tels qu'observés par le lidar Cats situé sur la Station spatiale internationale. Les cycles diurnes sont calculés sur les étés 2015-2017 au-dessus des océans et restreints aux zones de subsidence de grande échelle. Figure modifiée tirée de Chepfer et al. (2019). 
L'instrument Scarab mesure des flux sortants au sommet de l'atmosphère dans les domaines solaire (gamme de 0,2 à $4 \mu \mathrm{m}$ ) et de l'infrarouge (4 à $200 \mu \mathrm{m}$, voir tableau 1) à différents moments du cycle diurne, ceci grâce à l'orbite très particulière du satellite Megha-Tropiques, venant ainsi compléter la documentation offerte par les orbites héliosynchrones telles celle de l'A-Train. La figure 4 illustre la méthodologie utilisée. Dans chaque fauchée de Scarab, les systèmes convectifs identifiés par Toocan sont projetés et les pixels Scarab ainsi désignés sont alors attribués à un système convectif dont on connaît la phase dans le cycle de vie normalisé (entre 1 et 10). Le panel du haut de la figure 4 met en lumière un certain nombre de précautions nécessaires : d'une part, les produits de flux instantanés sont fournis à une résolution fixe de $0,5^{\circ} \times 0,5^{\circ}$; on observe ainsi que certains de ces points de grille ne sont que partiellement colocalisés avec un système convectif détecté ; d'autre part, bien que la fauchée de l'instrument mesure plus de $2000 \mathrm{~km}$, certains systèmes ne sont que partiellement échantillonnés. Ces deux considérations amènent à une plus forte incertitude sur les cycles de vie composite pour les début et fin de vie comme montré sur la figure 4 .

Pour chaque système convectif colocalisé, la valeur moyenne de ses propriétés radiatives est moyennée avec celles des autres systèmes à la même étape. Bien que les observations soient instantanées, ceci permet une vision complète du cycle de vie comme illustré en figure 4 . On voit alors pour la région considérée (ici l'océan Pacifique) que les systèmes convectifs sont brillants au début de leur cycle de vie et que la capacité à réfléchir le rayonnement dans le visible diminue dans la deuxième moitié de leur vie. Dans l'infrarouge, on observe que les flux émis ont leur minimum dans la première moitié du cycle de vie. Ce comportement se retrouve d'une région géographique à une autre, mais des disparités dans les amplitudes et les ordres de grandeur existent, montrant l'importance de l'environnement dans lequel ces systèmes se développent. Ces nouvelles caractéristiques des systèmes tropicaux permettent d'offrir une contrainte observationnelle intéressante pour le développement de la représentation paramétrique de la convection dans les modèles du système Terre ou pour évaluer la nouvelle génération de modèles de climat qui représentent explicitement les systèmes convectifs.
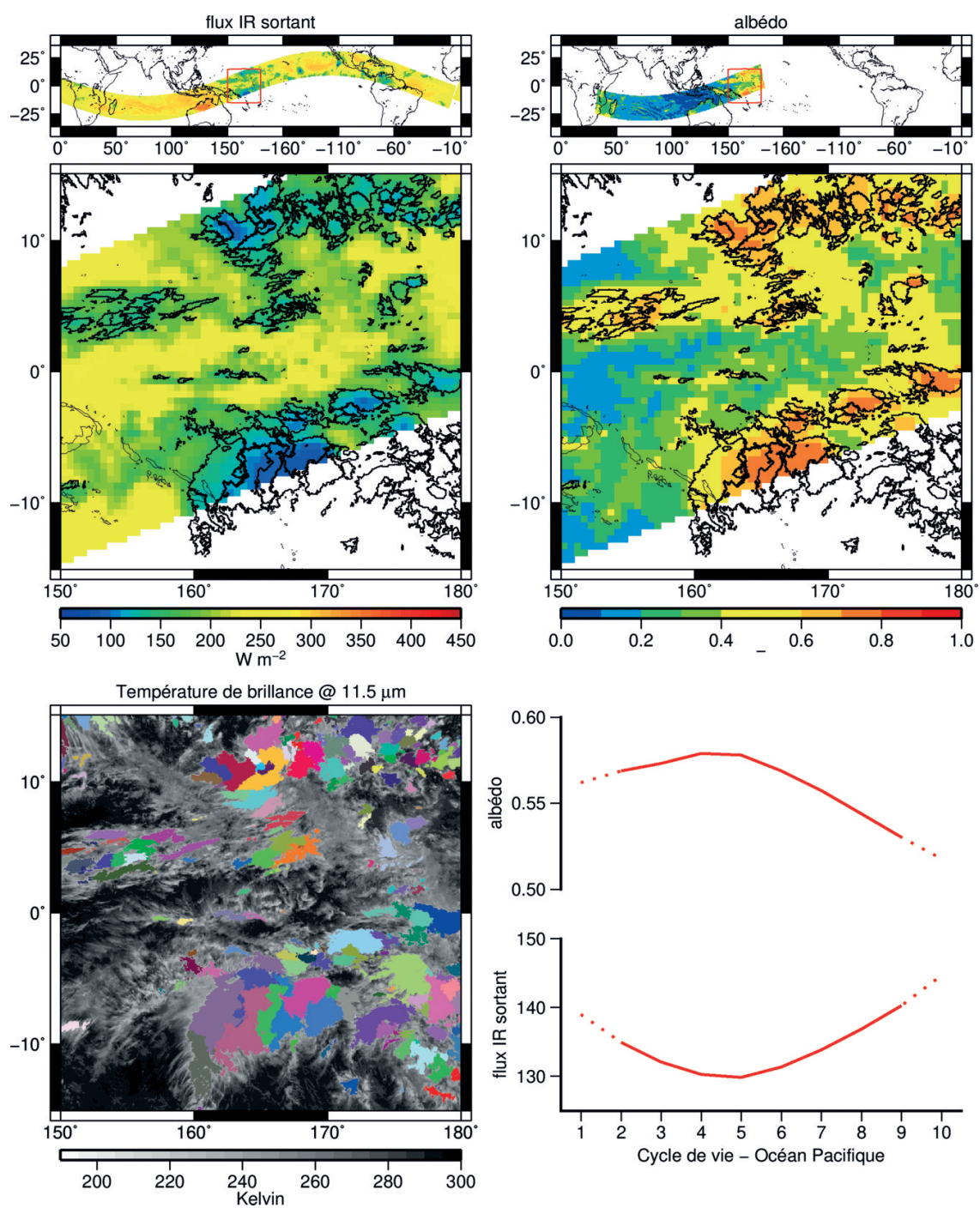

Figure 4. En haut, carte du flux sortant instantané au sommet de l'atmosphère dans l'infrarouge (noté flux IR sortant, à gauche) et de l'albédo (à droite) estimé à partir les mesures de l'instrument Scarab. Un zoom pour chaque grandeur à l'intérieur du carré rouge est présenté. Les contours noirs représentent l'enveloppe des systèmes convectifs identifiés dans l'image infrarouge (en bas à gauche), où ces mêmes systèmes sont montrés avec une couleur différente pour chaque objet. La partie basse droite montre l'évolution composite de ces propriétés radiatives au cours du cycle de vie des systèmes convectifs (moyennés pour les heures où le soleil est proche du zénith pour l'albédo) pour les systèmes échantillonnés au dessus de l'océan Pacifique (hors terres émergées). Le début et la fin du cycle de vie sont affichés en pointillés à cause d'un degré de confiance inférieur pour ces étapes.

\section{Pluie et hydrométéorologie}

L'augmentation des risques hydrométéorologiques et des dégâts humains et matériels qui en découlent pour les populations de la zone intertropicale est attestée par plusieurs études (Winsemius et al., 2015 ; Jongman, 2018) et confirmée sur le terrain par les organisations d'aide au développement. Les causes de ces inondations récurrentes restent cependant en partie incomprises. Les processus physiques qui sous-tendent la relation entre pluie et débit sont complexes, non linéaires, fortement dépendants des échelles spatio-temporelles considérées et influencés par des facteurs anthropiques comme l'occupation du sol.
Le volet « hydrométéorologie » de Megha-Tropiques analyse l'apport des produits pluviométriques satellitaires à l'analyse des inondations tropicales et de leur causalité. Un préalable est de quantifier la précision de ces produits, leurs incertitudes. L'évaluation du produit Tapeer (Tropical Analysis of Precipitation with an Estimation of ERrors) par rapport à des réseaux de pluviomètres de référence et les intercomparaisons avec d'autres produits satellitaires de 1'ère GPM ont démontré les très bonnes performances de Tapeer, un des meilleurs produits de sa génération, notamment en Afrique de l'Ouest (Gosset et al., 2018 ; Roca et al., 2018). 
Une originalité de l'approche Tapeer est de fournir une barre d'erreur à la pluie journalière qu'estime le produit. En hydrologie, cette variable permet d'analyser la propagation des incertitudes du forçage pluviométrique dans la prévision des débits et des inondations. Ces études hydrométéorologiques se sont concentrées sur le fleuve Niger et le problème de la crue « rouge » à l'origine d'inondations récurrentes à Niamey au cours des dernières années. Ce phénomène est directement lié à l'impact des systèmes convectifs, pendant le cœur de la saison des pluies, sur trois petits affluents situés en rive droite du fleuve Niger, en amont de Niamey (figure 4). La zone contributive s'étend sur une centaine de milliers de kilomètres carrés (quelques pixels Tapeer), ce qui exige une bonne localisation et une quantification de la pluviométrie associée à ces systèmes convectifs de méso-échelle.

Une modélisation hydrologique permettant de prendre en compte la variabilité de la pluie a été mise en place pour le fleuve Niger (Fleichmann et al.,
2018). Le produit Tapeer est utilisé en entrée du modèle pour analyser le rôle de la pluie dans la genèse des inondations et notre capacité à reproduire ces phénomènes dans les simulations numériques ces phénomènes. Des ensembles de scénarios de pluie en entrée du modèle hydrologique sont produits à partir de la barre d'erreur Tapeer et de différentes hypothèses sur la structure spatio-temporelle de l'erreur. Le résultat est un ensemble de simulations, dont la dispersion traduit l'effet des incertitudes pluviométriques sur la précision des débits simulés et sa sensibilité à la structure de ces erreurs (corrélation spatiotemporelle; localisation dans le bassin). Les simulations sur le fleuve Niger confirment en outre les bonnes performances de Tapeer en zone sahélienne et sa pertinence pour des applications hydrologiques comme illustré sur la figure 5 .

Ces travaux ont fait 1'objet d'un scénario de démonstration dans le cadre du projet de Space Climate Observatory (SCO), un programme international de suivi satellitaire des impacts de la variabilité climatique, lancé par le Cnes en 2019. L'approche est en cours de généralisation sur d'autres bassins tropicaux, en Amérique du Sud et sur le fleuve Congo - un bassin particulièrement mal instrumenté malgré les enjeux que suscitent l'hydrométéorologie de ce fleuve africain majeur et sa sensibilité aux variations climatiques (impact des extrêmes ; activités socio-économiques directement liées au débit et à la ressource hydrique). De nouveaux développements sont en cours pour améliorer Tapeer en tirant mieux partie de la collaboration avec le programme GPM, en intégrant les produits de niveau 2 délivrés par la Nasa sur l'ensemble des imageurs et sondeurs (dont Saphir) de la constellation.

La démarche d'hydrométéorologie spatiale initiée dans Megha-Tropiques nourrit une réflexion plus large sur l'utilisation complémentaire de différentes missions satellitaires en hydrologie ; elle s'intègre dans la dynamique scientifique initiée avec la future mission altimétrique haute résolution globale Swot (Surface Water a) Débit Garbe Kourou (sim et obs)

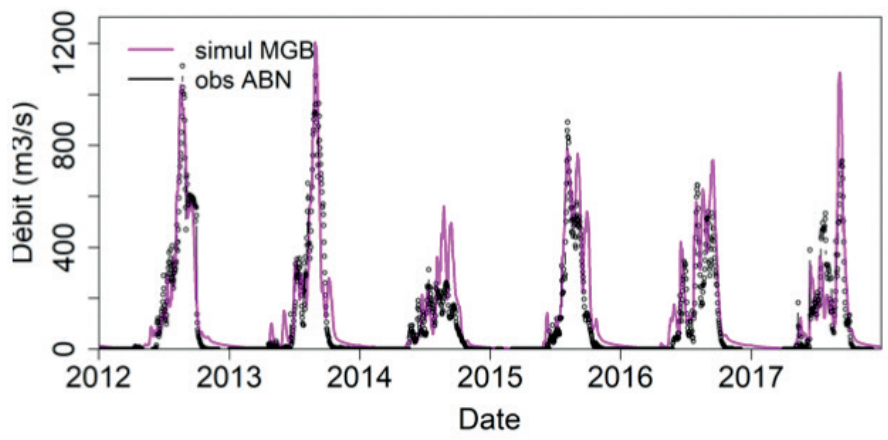

c) Pluie Erreur Standard (mm/jour) 2016-09-23

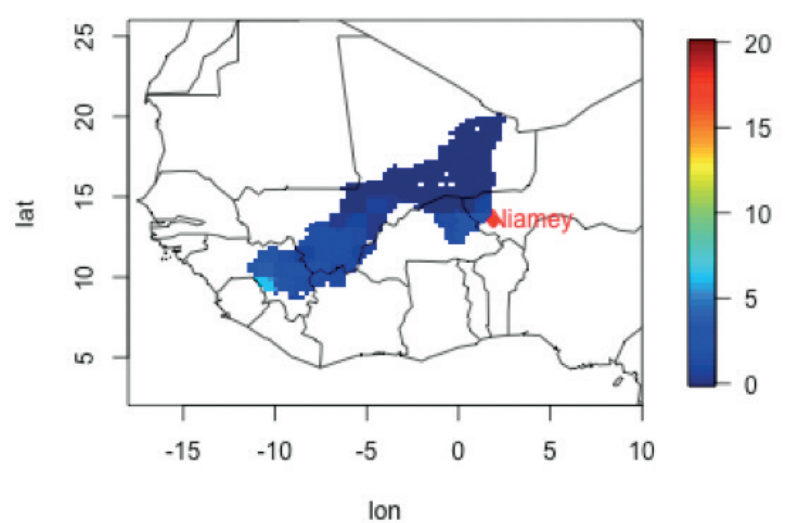

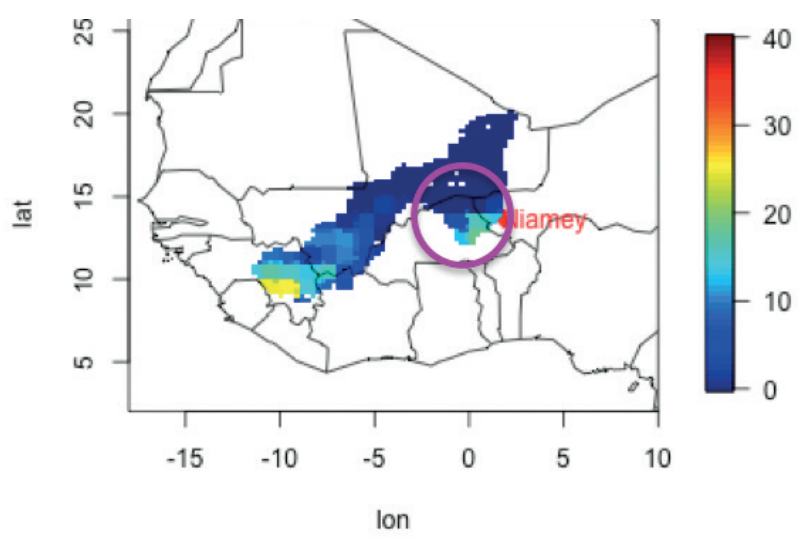

d) Niamey : débit simulé

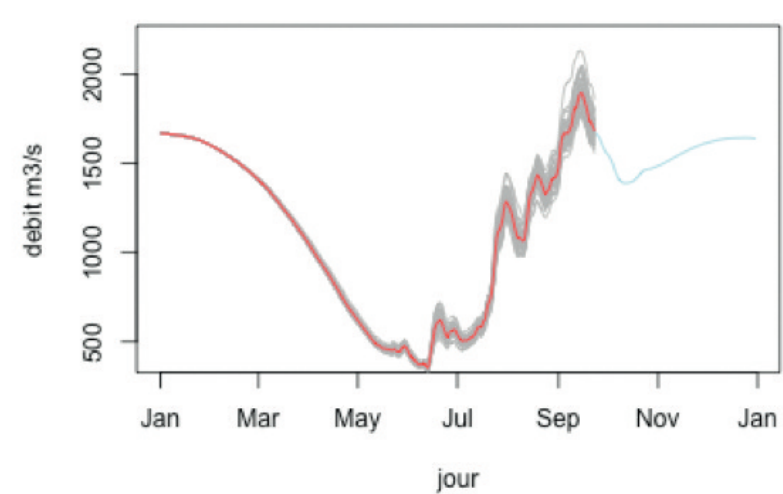

Figure 5. Simulation du débit du fleuve Niger et de ses affluents à partir d'un modèle hydrologique (MGB) et du produit Tapeer en forçage : a) débit simulé (cyan) et observé (noir) depuis 2012 à la station Garbe-Kourou, un des petits affluents responsables de la crue " rouge " et de ses inondations : b) la pluie journalière estimée par Tapeer (mm) sur le bassin du Niger (ici, le 23/09/2016), le cercle cyan représente la zone des affluents de rive droite responsables des inondations ; c) L'erreur standard de Tapeer $(\mathrm{mm})$ utilisée pour réaliser des simulations ensemblistes ; d) débit simulé à Niamey en 2016, avec, en rouge, la simulation sans erreur sur Tapeer et en gris la dispersion des ensembles. 
Ocean Topography) qui mesurera les variations d'eau de toute rivière de largeur supérieure à $100 \mathrm{~m}$, offrant de nouvelle perspectives pour l'hydrométéorologie des bassins peu jaugés.

Les illustrations des résultats scientifiques ci-dessus mettent principalement en avant la contribution française, mais ne doivent pas faire oublier les travaux de nos collègues d'Inde et d'autres pays qui se sont, eux aussi, appropriés avec succès les observations de Megha-Tropiques pour leurs problématiques scientifiques. Aussi, de nombreux travaux de télédétection ont pu voir le jour autour de Megha-Tropiques, que nous avons délibérément omis de lister ici, mais qui contribuent à l'exploitation des autres missions, micro-ondes en particulier, passées ou futures.

\section{Les prévisions météorologiques opérationnelles}

Bien que qualifiée de mission recherche, le Cnes et l'Isro ont doté (sous l'impulsion des scientifiques) de capacités quasi opérationnelles la mission Megha-Tropiques, tant par le maintien d'un segment sol primaire efficace à Bangalore sous la responsabilité de l'Isro que par l'ajout de stations de réception tropicales opérées par le Cnes. Ainsi, les données de l'instrument Saphir sont recueillies et transmises à l'Eumetsat pour redistribution vers les centres météorologiques opérationnels en temps réel depuis mai 2014.

\section{Un impact significatif sur les prévisions tropicales}

Plusieurs études ont été menées ces dernières années afin d'exploiter le contenu en information des observations de l'instrument Saphir pour la prévision numérique du temps. Les six canaux de l'instrument Saphir sont en effet sensibles à la fois à l'humidité troposphérique et à la présence d'eau condensée au sein des nuages, notamment sous forme solide. Des travaux ont été conduits grâce à des systèmes de prévision à l'échelle globale (par exemple, Chambon et al., 2015) et à des systèmes de prévision régionaux (par exemple, Guerbette et al., 2016). En particulier, les systèmes globaux étant souvent pourvus de systèmes d'assimilation variationelle à quatre dimensions, ces derniers ont pu pleinement tirer parti de la revisite fréquente de Saphir. En effet, un système d'assimilation variationelle à quatre dimensions a pour objectif de trouver la meilleure trajectoire du modèle de prévision sur une fenêtre de temps donnée. Cette trajectoire se retrouve être particulièrement bien contrainte grâce à l'orbite de MeghaTropiques.

En zone de ciel clair, il a été montré que les six canaux de Saphir apportent chacun une information pertinente afin d'améliorer les prévisions d'humidité, mais aussi de vents dans les Tropiques, et ce jusqu'à plusieurs jours d'échéance. Ces apports se chiffrent en quelques pourcents de réduction d'erreur sur certains paramètres prévus, ce qui peut paraître peu, mais est en fait capital pour un système de prévision du temps résilient à la perte de certains capteurs. Une étude récente a encore montré la place unique que Saphir occupe aujourd'hui dans un système de prévisions numériques à l'état de l'art tel que celui du Met Office ${ }^{6}$, avec des impacts sur les prévisions quasiment aussi importantes que quatre sondeurs de vapeur d'eau de même catégorie (MHS), en orbite héliosynchrone (Migliorini et Candy, 2019). En zone nuageuse, plusieurs démonstrations de

6. Service météorologique national britannique. de l'analyse. 1'utilité des observations de Saphir ont été menées. Les travaux conduits par le Centre européen pour les prévisions météorologiques à moyen terme (CEPMMT) sur l'assimilation d'observation des nuages en présence de précipitations sous forme solide pour les sondeurs AMSU-B et MHS ont pu être adaptés sur le sondeur Saphir (Chambon et Geer, 2017). Ces travaux reposent sur des développements en matière de transfert radiatif et l'utilisation de propriétés radiatives avancées pour l'eau dans sa phase solide (Geer et Baordo, 2014). Ils reposent également sur des développements en matière de spécification d'erreurs d'observation en ciel nuageux et pluvieux permettant d'améliorer le caractère gaussien des écarts entre le modèle de prévision et les observations, et donc l'optimalité

En ce qui concerne la prévision cyclonique, l'apport des observations de Saphir en ciel nuageux a pu être quantifié grâce au système de prévision à échelle mondiale de Météo-France Arpege. Les figures 6 et 7 montrent cet apport d'une part sur un exemple de cyclone et d'autre part d'un point de vue statistique sur un ensemble de 432 prévisions cycloniques. Pour le cas montré du cyclone Shanshan,

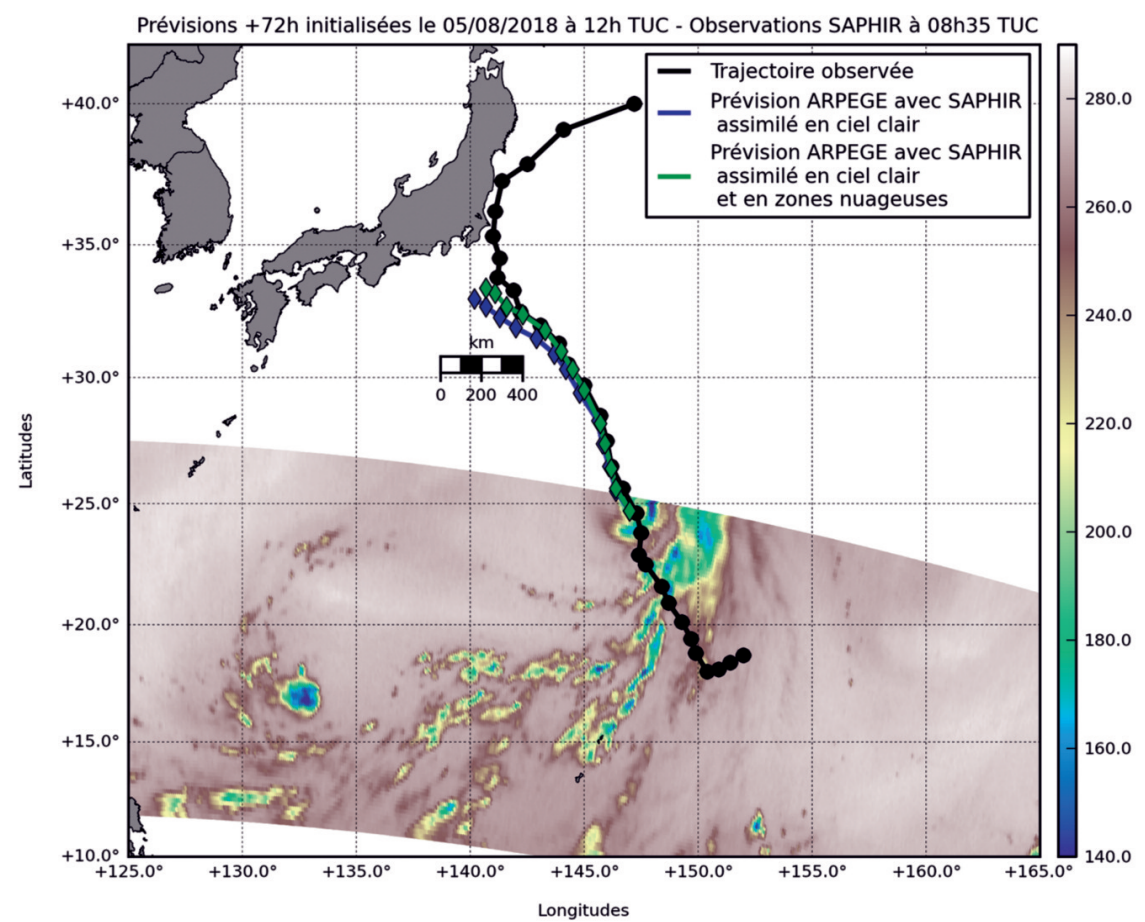

Figure 6. Position de l'œil du cyclone Shanshan (bassin du Pacifique Nord-ouest), toutes les 6 heures (courbe noire). Exemple de trajectoires prévues par le modèle Arpege jusqu'à 72 heures d'échéance, avec (courbe verte) et sans (courbe bleue) observations nuageuses de Saphir dans le système d'assimilation. 


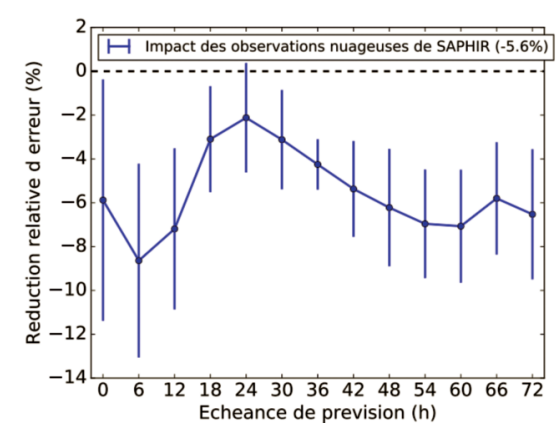

Figure 7. Statistiques de réduction d'erreur de prévision de trajectoires pour un échantillon de 16 cyclones (Beryl (ATL), Chris (ATL), Maria (NWP), Fabio (NEP), Ampil (NWP), Wukong (NWP), Shanshan (NWP), Jongdari (NWP), Hector (NEP), John (NEP), Florence (ATL), Helene (ATL), Isaac (ATL), Kirk (ATL), Michael (ATL), Jebi (NWP), 432 prévisions) sur trois bassins différents, réduction amenée par I'assimilation d'observations nuageuses de Saphir dans le système d'assimilation du modèle Arpege.

l'assimilation des observations Saphir conduit à une réduction de l'erreur sur la position de l'œil de plus de $100 \mathrm{~km}$ à 72 heures d'échéance. D'un point de vue statistique, les prévisions de trajectoires sont améliorées d'environ 6 \% grâce à Megha-Tropiques. Deux régimes d'amélioration sont visibles sur la figure 7 : la première amélioration est d'environ $9 \%$ en début d'échéance qui correspond aux cas cycloniques bien observés par Saphir ; la seconde est d'environ $6 \%$ et apparaît entre 30 et 72 heures. Ce second régime correspond aux cas cycloniques moins bien observés par Megha-Tropiques, mais qui bénéficient tout de même des meilleures prévisions de vents tropicaux amenées par Saphir. Ces travaux de recherches devraient être portés vers les chaînes opérationnelles de Météo-France dans les années à venir.

\section{Un succès planétaire}

Grâce à la diffusion en temps réel des données mise en place par le Cnes, l'Isro et Eumetsat, huit centres opérationnels assimilent aujourd'hui les observations de Saphir. Dans la foulée des efforts de Météo-France, ces assimilations opérationnelles ont démarré dès 2015 à l'Agence météorologique japonaise, ainsi qu'à Météo-France. Le Met Office a par la suite activé cette assimilation en 2016. Les travaux du Joined Center for Satellite Data Assimilation (JCSDA) au sein du système d'assimilation du modèle américain GFS (Global Forecast System) de la Noaa (National Oceanic and Atmospheric Administration) a ensuite permis aux deux centres américains, le Ncep (National Center for Environmental Prediction) et le FNMOC (Fleet Numerical Meteorology and Oceanography Center) d'intégrer ces données à leurs chaînes de traitement. Enfin, le CEPMMT ainsi que l'Agence météorologique coréenne ont également débuté leur assimilation des observations de Saphir en 2017. Le tableau 2 résume la liste des différents centres de prévision numérique du temps assimilant les données de Saphir, en ciel clair ou bien en ciel clair et ciel nuageux. L'utilisation intensive des observations de Megha-Tropiques dans différentes chaînes de traitement opérationnelle de par le monde est un bon indicateur de la qualité des instruments et des systèmes de traitement et de diffusion des données qui ont été mis en place par les deux agences spatiales.

\section{Conclusions}

\section{En quelques mots}

Après un début d'opération compliqué et une accumulation de retards sur la disponibilité des observations et des produits, la démonstration de faisabilité de la mission Megha-Tropiques a pu finalement être apportée, confirmant l'esprit visionnaire de ses inventeurs ! La perte de l'instrument Madras a été compensée par l'utilisation décuplée de Saphir et a permis de soutenir l'étude de nos thèmes scientifiques. Ces observations nouvelles ont ainsi pu, entre autres, enrichir notre compréhension des relations nuages et humidité dans l'atmosphère tropicale, documenter l'évolution des propriétés radiatives des systèmes convectifs tropicaux au cours de leur cycle de vie et contribuer à préciser les raisons des modifications des crues au Niger. La prévision numérique du temps n'est pas en reste, avec des résultats impressionnants quant à l'amélioration de la qualité de la prévision cyclonique grâce à l'assimilation des observations de Saphir.

\section{Le futur}

$\mathrm{Au}$ moment où les derniers rapports des agences sur la mission indiquent une forte dégradation concernant la disponibilité des observations de MeghaTropiques, il est intéressant de se tourner vers le futur. Bien qu'il soit trop tôt pour synthétiser l'héritage de la mission, on peut souligner que, tant sur le plan des investissements algorithmiques qu'instrumentaux, les efforts autour de Megha-Tropiques vont être capitalisés. Ainsi, le Climate Monitoring SAF d'Eumetsat implémente à l'heure actuelle une version climatique globale (et non plus tropicale) de nos estimations de précipitation s'appuyant sur l'utilisation de la constellation microondes. De même, les développements instrumentaux de Madras ont nourri l'élaboration du radiomètre microondes à balayage conique à bord de la série des satellites MetOpSG d'Eumetsat. L'intérêt de l'orbite originale de la mission a ouvert la voie au projet Tropics, une constellation de nanosatellites $(10 \mathrm{~cm} \times 10 \mathrm{~cm} \times 30 \mathrm{~cm})$ en orbite inclinée sur l'équateur en cours de réalisation par la Nasa, et le Cnes explore depuis peu des concepts d'instrument Saphir-nouvelle génération... L'observation spatiale du cycle de l'eau tropicale à de beaux jours devant elle!

Tableau 2. Liste des centres météorologiques utilisant Saphir en temps réel.

\begin{tabular}{lll} 
Centre de prévision numérique du temps & Observations assimilées & Référence bibliographique \\
$\begin{array}{l}\text { Centre européen pour les prévisions } \\
\text { météorologiques à moyen terme (CEPMMT) }\end{array}$ & Ciel clair et ciel nuageux & Chambon et Geer (2017) \\
\hline $\begin{array}{l}\text { Fleet Numerical Meteorology and } \\
\text { Oceanography Center (FNMOC) - U.S. Navy }\end{array}$ & Ciel clair & $\begin{array}{l}\text { Jones et al. (2017) } \\
\text { Karpowicz et al. (2017) }\end{array}$ \\
\hline Japanese Meteorological Agency (JMA) & Ciel clair & Jones et al. (2017) \\
\hline Joint Center for Satellite Data & Ciel clair & Lee et al. (2018) \\
Assimilation (JCSDA) & & Ciel clair \\
\hline Korean Meteorological Administration & Ciel clair & Jones et al. (2017) \\
\hline Météo-France & Ciel clair & Doherty et al. (2018) \\
\hline National Center for Environmental Prediction (Ncep) & Ciel clair & \\
\hline Met Office & &
\end{tabular}




\section{Remerciements}

De nombreuses personnes ont contribué au succès de la mission au cours des 25 dernières années. Les auteurs sont très reconnaissants à Michel Desbois, qui a initié et dirigé la mission MeghaTropiques de 1993 à 2007. Nous remercions également feu Michel Viollier pour son importante contribution aux développements scientifiques de Scarab. F. Aires, M. Alcoba, M. Capderou, O. Chomette, C. Dufour L. Eymard, I. Jobard, S. Kacimi, P.E. Kirstetter, A. Lifermann, J.-F. Mahfouf, A. Martini, D. Renaut, J.-L. Renaud, G. Sèze, A. Szantai, N. Viltard sont tous chaleureusement remerciés pour leur contribution utile au succès de l'exploitation scientifique à différentes étapes de son développement. Un grand nombre de programmeurs sous contrat à court terme nous ont considérablement aidés à finaliser les algorithmes et les produits et nous sommes reconnaissants de leur contribution à la mission. L'exploitation scientifique de la mission a bénéficié en outre des discussions stimulantes tenues dans le cadre du groupe de recherche Insu Megha-Tropiques. Les auteurs remercient en outre N. Karouche et T. Baroso, ainsi que M. Dejus, responsables successifs de projet au Cnes, pour avoir permis à la mission de devenir une réalité. L'équipe Espri de l'Institut PierreSimon Laplace a efficacement hébergé la phase de développement des algorithmes scientifiques ainsi que la phase d'exploitation des données. Le groupe Icare (une des composantes du nouveau pôle national Aeris) est également reconnu pour avoir réalisé le segment sol scientifique de la mission. Les données Megha-Tropiques sont disponibles sur http://www.icare. univ-lille1.fr/mt. Le Cnes et le CNRS sont remerciés pour leur soutien financier à l'activité scientifique de la mission.

\section{Bibliographie}

Bouniol D., Roca R., Fiolleau T. Poan E., 2016. Macrophysical, microphysical, and radiative properties of tropical mesoscale convective systems along their life cycle. J. Clim., 29, 33533371. doi: 10.1175/JCLI-D-15-0551.1

Capderou M., 2017. Les orbites des satellites météorologiques. La Météorologie, 97, 94-101. doi: 10.4267/2042/62172

Chambon P., Meunier L., Guillaume F., Piriou J., Roca R., Mahfouf J., 2015. Investigating the impact of the water vapour sounding observations from SAPHIR on board Megha-Tropiques for the ARPEGE global model. Q. J. R. Meteorol. Soc., 141, 1769-1779. doi: 10.1002/qj.2478

Chambon P., Geer A.J., 2017. All-sky assimilation of Megha-Tropiques/SAPHIR radiances in the ECMWF numerical weather prediction system. European Centre for Medium-Range Weather Forecasts Technical Memorandum 802. Disponible à l'adresse: https://www.ecmwf.int/node/17253. doi: 10.21957/4h085eb9

Chepfer H., Brogniez H., Noël V., 2019. Diurnal variations of cloud and relative humidity profiles across the tropics, Nature Scientific Report, sous presse..

Desbois M., Capderou M., Eymard L., Roca R., Viltard N., Viollier M., Karouche N., 2007. Megha-Tropiques : un satellite hydrométéorologique franco-indien. La Météorologie, 57, $19-27$. doi: $10.4267 / 2042 / 18185$

Doherty A, Indira Rani S., Newman S., Bell W., 2018. Benefits of assimilating SAPHIR observations on analysis and forecasts of tropical fields in the Met Office global model. Q. J. R. Meteorol. Soc., 144, 405-418. doi: 10.1002/qj.3258

Fiolleau T., Roca R., 2013. An algorithm for the detection and tracking of tropical mesoscale convective systems using infrared images from geostationary satellite. IEEE Transactions on Geoscience and Remote Sens., 51, 4302 - 4315. doi: 10.1109/TGRS.2012.2227762

Fleischmann A., Siqueira V., Paris A., Collischonn W., Paiva R., Pontes P., Crétaux J.-F., Bergé-Nguyen M., Biancamaria S., Gosset M., Calmant S., Tanimoun B., 2018. Modelling hydrologic and hydrodynamic processes in basins with large semi-arid wetlands. J. Hydrology, 61, 943-959. doi: 10.1016/j.jhydrol.2018.04.041

Geer A.J., Baordo F., 2014. Improved scattering radiative transfer for frozen hydrometeors at microwave frequencies. Atmos. Meas. Tech., 7, 1839-1860.

Gosset M., Alcoba M., Roca R., Cloché S., Urbani G., 2018. Evaluation of TAPEER daily estimates and other GPM era products against dense gauge networks in West Africa, analysing ground reference uncertainty. O. J. R. Meteorol. Soc., 144, 255-269. doi: 10.1002/qj.3335

Guerbette J., Mahfouf J.-F., Plu M., 2016. Towards the assimilation of all-sky microwave radiances from the SAPHIR humidity sounder in a limited area NWP model over tropical regions. Tellus, 68A, 28620. doi: 10.3402/tellusa.v68.28620

Hou A.Y., Kakar R.K., Neeck S., Azarbarzin A.A., Kummerow C.D., Kojima M., Oki R., Nakamura K., Iguchi T., 2014. The Global Precipitation Measurement (GPM) mission. Bull. Am. Meteorol. Soc., 95, 711-722. doi: 10.1175/BAMS-D-13-00164.1

Houze R.A. Jr, 2018. 100 Years of research on mesoscale convective systems. AMS monograph, 17.1-17.53. doi: 10.1175/AMSMONOGRAPHS-D-18-0001

Jones E.E., Garrett K., Boukabara S.A., 2017. Assimilation of Megha-Tropiques SAPHIR observations in the NOAA global model. Mon. Weather Rev., 145, 3725-3744. doi: 10.1175/MWR-D-16-0148.1

Jongman B., 2018. Effective adaptation to rising flood risk. Nat. Commun., 9, 1986. doi: 10.1038/s41467-018-04396-1

Karpowicz B., Ruston B., Swadley S., 2017. Microwave radiance assimilation at NRL: Advanced techniques, developments and future sensors. 21 st International TOVS Study Conference. Darmstadt, Germany, 29 November-5 December 2017. Disponible à l'adresse http://cimss.ssec.wisc.edu/itwg/itsc/itsc21/program/posters/4p.05 karpowicz.pdf

Migliorini S., Candy B., 2019. All-sky satellite data assimilation of microwave temperature sounding channels at the Met Office. 0. J. R. Meteorol. Soc., 145, 867-883. doi: 10.1002/qj.3470

Lee S., Chun H.-W., Song H.-J., 2018. Impact of SAPHIR data assimilation in the KIAPS global numerical weather prediction system. Atmosphere, 28, 141-151. doi: 10.14191/ATMOS.2018.28.2.141

Roca R., Brogniez H., Chambon P., Chomette 0., Cloché S., Gosset M.E., Mahfouf J.-F., Raberanto P., Viltard N., 2015. The Megha-Tropiques mission: a review after three years in orbit. Front. Earth Sci., 3, 17

Roca R., Fiolleau T., Bouniol D., 2017. A Simple model of the life cycle of mesoscale convective systems cloud shield in the Tropics. J. Clim., 30, 4283-4298. doi: 10.1175/JCLI-D-160556.1

Roca R., Taburet N., Lorant E. et al., 2018. Quantifying the contribution of the Megha-Tropiques mission to the estimation of daily accumulated rainfall in the Tropics. O. J. R. Meteorol. Soc., 144, 49-63. doi: 10.1002/qj.3327X

Sherwood S., Roca R., Weckwerth T., Andronova N., 2010. Tropospheric water vapor, convection and climate. Rev. Geophys., 48, RG2001. doi: 10.1029/2009RG000301

Winsemius H.C., Jongman B., Veldkamp T.I.E., Hallegatte S., Bangalore M., Ward P.J., 2015. Disaster risk, climate change, and poverty: assessing the global exposure of poor people to floods and droughts. Policy Research working paper No. WPS 7480. Washington, D.C.: World Bank Group. Disponible à I'adresse http://documents.worldbank.org/curated/en/965831468189531165/Disaster-risk-climate-change-and-poverty-assessing-the-global-exposure-of-poor-people-to-floods-and-droughts 